(LETTER TO EDITOR)

\title{
Antimicrobial resistant superbug warning watch: a newly emerged Klebsiella pneumoniae and Mycoplasma genitalium joining the group
}

\author{
Eissa Mostafa*
}

Microbiology and Immunology Department, Cairo University, Cairo, Egypt.

Publication history: Received on 14 December 2018; revised on 25 February 2019; accepted on 10 June 2019

Article DOI: https://doi.org/10.30574/wjarr.2019.2.2.0002

\section{Dear Sir,}

Spreading of drug resistance has become a commonly reported phenomenon. The list of microorganisms that are joining the group of superbugs is continuously increasing [1]. The fast and efficient adaptability mechanism of microbial cells is the essence of their ability to survive unfavorable environmental conditions which may be biological, physical and/or chemical [2].

One of the Gram-negative bacteria that may turn into a harmful organism is known as Klebsiella pneumoniae. K. pneumoniae is a normal intestinal flora in human, but it can cause dangerous infections if it reaches other parts of the body such as lungs, blood, or wounds. The risk of infection from this microbe may increase with people with specific health conditions or troubles ex. cancer, lung diseases, alcoholism, liver problems and kidney failure. This microorganism may turn into a superbug that can resist the most commonly known antibiotics [3]. Recently fatal cases have been reported in hospitals where hyper-virulent and hyper-resistant strains of $K$. pneumoniae were isolated [4, 5]. Even some strains have been shown to be almost resistant to all antibiotics, others are flesh eating and may cause blindness and meningitis [The wire, https://thewire.in/health/superbug-india, Last accessed on 13/12/2018; Telegraph, https://www.telegraph.co.uk/news/2018/12/07/dangerous-new-superbug-confirmed-india-antibioticresistance/, Last accessed on 13/12/2018]. The frequency of this trend of resistance and virulence seems to be rising with time globally.

Another warning signal comes for Mycoplasma genitalium (MG), a sexually transmitted disease (STD) that could be a source of hazard as an untreatable disease [6]. While this smallest self-replicating microorganism was discovered in 1981, the seriousness of its impact on the human genitourinary tract has recently brought attention as a public health problem [7]. The developed countries have started to list this underestimated microbe as an emerging issue that should not be overlooked, otherwise drug resistance problem to the first- and second-defense lines antibiotic treatment may be exacerbated during the next decade [Contagion, https://www.contagionlive.com/news/uk-health-officials-aim-tostop-the-emerging-superbug-mycoplasma-genitalium, Last accessed on 13/12/2018].

\section{Conclusion}

Microbial resistance to antimicrobials is a continuous progressive threat that threatens human health and life. In the absence of effective recording and tracking system in many developing countries, the actual rate of resistance emergence may be much higher than that is actually available in the database. While the list of superbugs is growing globally, the number of human population with the defected health system is still rising. Working on antibiotic treatment protocols only seems to be not enough to withhold the spreading of the drug resistance and extensive efforts are required worldwide to find new cures for infections to shift the balance towards susceptibility rather than resistance to avoid reverting back to the pre-antimicrobialera.

\footnotetext{
${ }^{*}$ Corresponding author

E-mail address: mostafaessameissa@yahoo.com
} 


\section{Compliance with ethical standards}

\section{Disclosure of conflict of interest}

The author declares that there is not any competing conflict of interest.

\section{References}

[1] Prestinaci F, Pezzotti P and Pantosti A. (2015). Antimicrobial resistance: a global multifaceted phenomenon. Pathogens and global health, 109(7), 309-18.

[2] Peterson JW. (1996). Medical Microbiology, Fourth edition. University of Texas Medical Branch at Galveston, Galveston, USA.

[3] What to know about the Klebsiella pneumoniae Superbug? What is Klebsiella pneumoniae Infection? [WebMD, https://www.webmd.com/a-to-z-guides/klebsiella-pneumoniae-infection\#1, Last accessed on 13/12/2018].

[4] The Hong Kong Polytechnic University, Newly emerged superbug discovered [ScienceDaily, www.sciencedaily.com/releases/2017/08/170831101508.htm, Last accessed on 12/12/2018].

[5] Gu D, Dong N, Zheng Z, Lin D, Huang M, Wang L, Chan EW, Shu L, Yu J, Zhang R and Chen S. (2018). A fatal outbreak of ST11 carbapenem-resistant hypervirulent Klebsiella pneumoniae in a Chinese hospital: a molecular epidemiological study. The Lancet infectious diseases, 18(1), 37-46.

[6] Kirby T. (2018). Mycoplasma genitalium: a potential new superbug. The Lancet Infectious Diseases, 18(9), 951952.

[7] Hughes G and Saunders J. (2018). Mycoplasma genitalium: the next sexually transmitted superbug?. BMJ, k4376.

\section{How to cite this article}

Eissa M. (2019). Antimicrobial resistant superbug warning watch: a newly emerged Klebsiella pneumoniae and Mycoplasma genitalium joining the group. World Journal of Advanced Research and Reviews, 2(2), 17-18. 